\title{
Nonlinear diffusion in magnetized discharges
}

\section{Francis F Chen}

Electrical Engineering Department, University of California, Los Angeles, CA 90095-1594, USA

Received 4 February 1998, in final form 9 April 1998

Abstract. In high-density gas discharges in which the electrons, but not the ions, are confined by a magnetic field, the radial density profile is often observed to have a triangular shape, as if the ionization were concentrated on axis. However, such profiles can also result from nonlinear diffusion, in which the diffusion coefficient varies inversely with density.

\section{Introduction}

Magnetically enhanced inductive discharges are often used to produce high-density plasmas for industrial purposes. Research on helicon discharges in uniform cylinders, for instance, is being pursued in many laboratories in order to study the behaviour of partially ionized plasmas with a DC magnetic field capable of confining the electrons but not strong enough to confine the ions. In such discharges, the radial density profile is sometimes observed to have a triangular shape [1]. A recent example of such a profile is shown in figure 1 [2]. If radial diffusion is controlled by diffusion in a background of neutrals, one would expect a convex density profile; for instance, a $J_{0}$ Bessel function if the ionization is proportional to electron density [3]. A concave or straight profile would suggest that ionization is heavily concentrated on axis, in contradiction to theoretical results on the coupling of helicon waves to Trivelpiece-Gould modes near the surface [4]. In this paper we explore the possibility that these strange profiles are caused by an unusual classical diffusion mechanism.

\section{Diffusion in intermediate magnetic fields}

Consider an infinitely long plasma column confined in a tube of radius $a$ with a coaxial DC magnetic field $\boldsymbol{B}_{0}$ of 50-1500 G magnitude. In this field, electrons will usually have Larmor radii $r_{L}$ much smaller than $a$, but heavy ions, such as argon, will have $r_{L} \gg a$. Ions will diffuse radially outward, impeded by collisions with the neutral gas; but they will also be impeded by collisions with electrons, since the electrons are restrained by the magnetic field and cannot easily move radially with the ions. The physical picture is that the slowly drifting ions are continuously bombarded by electrons moving along $\boldsymbol{B}_{0} \hat{\boldsymbol{z}}$. The momentum exchange is mostly in the $z$ direction, but the small amount of radial momentum that the ions lose to the electron 'rods' is what impedes ion loss. In principle, there should be an ambipolar

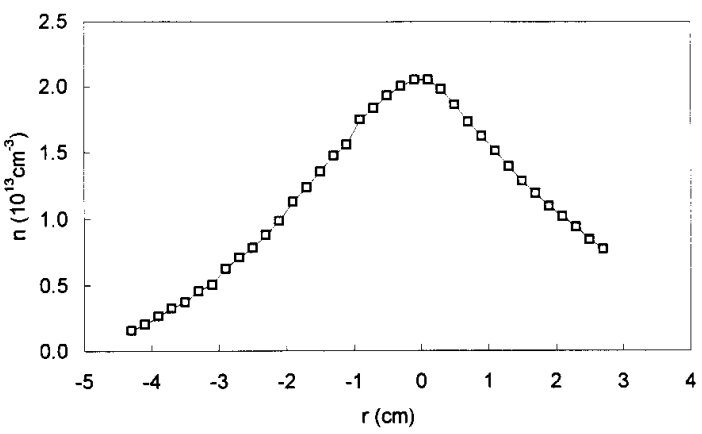

Figure 1. Typical 'triangular' density profile, with $a=5 \mathrm{~cm}$, $B_{0}=640 \mathrm{G}, p_{0}=3 \mathrm{mT}$ Torr of argon, $P_{r f}=1 \mathrm{~kW}$ at 13.56 $\mathrm{MHz}$ [2].

electric field slowing the radial ion motion, but in practice such fields are not usually observed. Radial variations in potential tend to be weak and of the wrong sign to give an ambipolar field [5]. Figure 2 shows a radial profile of plasma potential obtained from the inflection point of the $I-V$ characteristic of a fully RF-compensated Langmuir probe [2]. The plasma potential does not follow the density variation, and the radial $E$-field is too weak to affect the ion motions.

The absence of an inward pointing $E$-field is expected only if axial transport can be neglected, as in very long discharges. In the opposite limit of isotropic diffusion, one would expect the electrons to thermalize into a Boltzmann distribution $n_{e}=n_{0} \exp \left(e V / K T_{e}\right)$, so that the potential follows the density profile and the $E$-field points outwards. In the intermediate case of magnetized discharges of finite length, the short-circuit effect comes into play. In this mechanism, the sheath drop on the end wall terminating each magnetic field line can adjust itself to bleed off excess electrons on that field line (or to cure a deficit of electrons by erecting a higher Coulomb barrier) so as to maintain a self-consistent radial $E$-field. Though a detailed calculation is almost impossible because electron motions can also be 


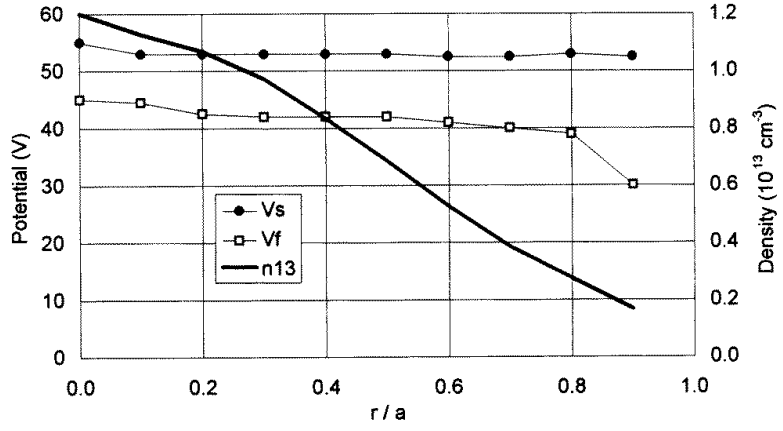

Figure 2. Radial profiles of density, space potential $V_{s}$, and floating potential $V_{f}$ for a $10 \mathrm{~cm}$ diameter discharge at $600 \mathrm{G}, 6 \mathrm{mTorr}$ and $1.5 \mathrm{~kW}$ at $13.56 \mathrm{MHz}$ [2].

affected by high-frequency instabilities, the result can be the near elimination of the radial $E$-field, as is observed. We therefore neglect the radial electric field from now on and consider the diffusion of ions in a background of neutrals and 'stiff' electrons.

The total ion collision frequency is given by

$$
v=v_{i e}+v_{i n}+v_{i i}
$$

where the last term represents ion-ion collisions, which can safely be neglected since there is no shear flow. The singly ionized ion-electron collision frequency $v_{i e}$ is given by Book [6] as

$$
v_{i e}=1.6 \times 10^{-9} n_{e} \ln \Lambda / A_{i} T_{e}^{1 / 2} E_{i}
$$

where $A_{i}$ is the ion mass number, $T_{e}$ is the electron temperature in $\mathrm{eV}, E_{i}$ is the ion energy and the Coulomb $\operatorname{logarithm} \ln \Lambda$ for these plasmas is about 10.7. For a Maxwellian ion distribution, we may approximate $E_{i}$ by $(3 / 2) T_{i}$.

The ion-neutral collision frequency $v_{i n}$ is dominated by charge exchange, the cross section for which is given for low energies by Sheldon [7]. Since this was found to agree with mobility data, we can also infer $v_{i n}$ from the measured mobility of $\mathrm{Ar}^{+}$in Ar, namely, $1.5 \mathrm{~cm}^{2} \mathrm{~V}^{-1} \mathrm{~s}^{-1}$ at 760 Torr and $296 \mathrm{~K}$ [8]. This yields

$$
v_{i n}=2.05 \times 10^{4} p_{0} \text { (mTorr) } .
$$

The critical density above which ion-electron collisions are dominant over ion-neutral collisions is therefore given by

$$
n_{\text {crit }}=2.05 \times 10^{4} \frac{p_{0}}{v_{i e} / n_{e}} .
$$

For $T_{e}=3 \mathrm{eV}$ and $T_{i}=0.0257 \mathrm{eV}$ in argon, this gives $n_{\text {crit }} \approx 10^{13} \mathrm{~cm}^{-3}$, a rather high value even with roomtemperature ions. However, at these densities the neutral pressure inside the plasma is reduced by the ion-pumping effect, which was inferred by Sudit and Chen [5] and has since been confirmed by direct measurement [9].

To estimate the ion pumping effect, consider a plasma of radius $a$ and length $L$, so that the volume $V$ is $V=$ $\pi a^{2} L$. Initially, gas is bled in at a flux $\Gamma_{i n}$ atoms $\mathrm{s}^{-1}$ and pumped out at a rate $S \mathrm{~cm}^{3} \mathrm{~s}^{-1}$, resulting in a fill pressure $p_{0}$ (mTorr) and a neutral density $n_{n 0}=p_{0} N_{0}$, where $N_{0}=3.3 \times 10^{13} \mathrm{~cm}^{-3}$ at $300 \mathrm{~K}$. After the discharge is struck, the neutrals inside the plasma are ionized, and ions with an average density $n_{i}$ are accelerated by the pre-sheath electric field toward the pump at one end of the discharge tube. We assume that the pump manifold is large enough to remove the recombining ions without appreciable refluxing of the neutrals, and we neglect the transit time of the accelerated ions. Next, we compute the neutral density $n_{n 1}$ in the chamber when the plasma is on. The flux of atoms leaving the plasma at both ends is $\mathrm{d} N_{i} / \mathrm{d} t=-2 \pi a^{2} \frac{1}{2} n_{i} c_{s}$, where $c_{s}$ is the ion sound speed $\left(K T_{e} / M\right)^{1 / 2}$ and $\frac{1}{2} n_{i}$ is the ion density at the sheath edge. Ions are created at the rate $\mathrm{d} N_{i} / \mathrm{d} t=\pi a^{2} L n_{e} n_{n 1} P_{i}$ where $P_{i} \equiv\langle\sigma v\rangle_{i o n}$ is the ionization probability, a sensitive function of $T_{e}$. Equating this to the loss rate, we find that $n_{e}=n_{i}=n$ cancels, and $n_{n 1}$ is given by

$$
n_{n 1}=c_{s} / L P_{i} .
$$

If $\Gamma_{i n}$ is not measured, we can find $n_{n 0}$ as follows. Neutrals are lost at the rate that ions are lost at the pump end of the tube:

$$
\Gamma_{\text {out }}=\pi a^{2} \frac{1}{2} n_{i} c_{s} .
$$

Equating this to $\Gamma_{i n}=S n_{n 0}$, we have

$$
n_{n 0}=\pi a^{2} \frac{1}{2} n_{i} c_{s} / S .
$$

The pressure reduction ratio is therefore given by (5) and (7) as

$$
\varepsilon \equiv \frac{n_{n 1}}{n_{0}}=\frac{2 S}{V n_{e} P_{i}} .
$$

Since the diffusion coefficient for unmagnetized particles is given by $D=K T / M v$, (2) leads to

$$
D_{i e}=8.4 \times 10^{19} T_{e}^{1 / 2} T_{i}^{2} / n_{e}
$$

while (3) gives (for argon)

$$
D_{\text {in }}=1.2 \times 10^{6} T_{i} / p_{0} .
$$

Substituting $\varepsilon p_{0}$ for $p_{0}$ in (10) and solving for $n_{e}$, we obtain

$$
n_{\text {crit }}=\left(1.4 \times 10^{17} T_{e}^{1 / 2} T_{i} S / V P_{i}\right)^{1 / 2} .
$$

For instance, for $T_{e}=3 \mathrm{eV}, T_{i}=0.1 \mathrm{eV}$ (assuming some heating by the electrons), $S=10^{5} \mathrm{~cm}^{3} \mathrm{~s}^{-1}$, and $a=5 \mathrm{~cm}$, $n_{\text {crit }}$ is $1.2 \times 10^{12} \mathrm{~cm}^{-3}$. As $T_{e}$ ranges from 2 to $5 \mathrm{eV}$, $n_{\text {crit }}$ changes from $5 \times 10^{12}$ to $4 \times 10^{11} \mathrm{~cm}^{-3}$, densities usually well exceeded in the experiments in question. We may therefore neglect diffusion via ion-neutral collisions.

\section{Radial profiles}

In the previous section we have tried to justify the neglect of $v_{i n}$; we now explore the consequences if this approximation is reasonable. With the use of (2) the ion diffusion coefficient is given by

$$
D=\frac{K T_{i}}{M v_{i e}} \equiv \frac{A}{n}
$$




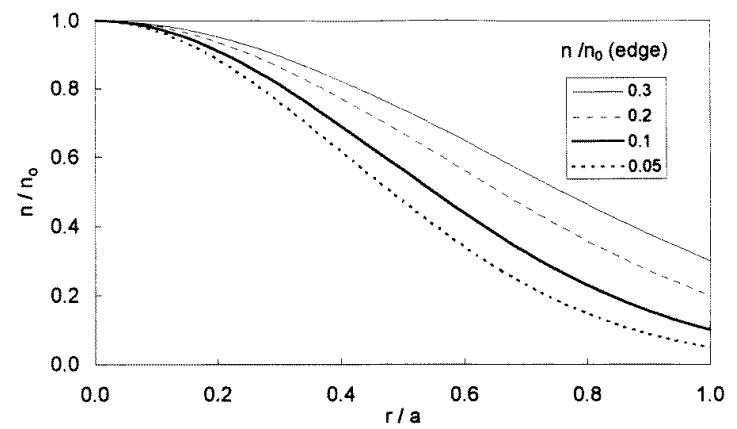

Figure 3. Computed density profiles for uniform ionization.

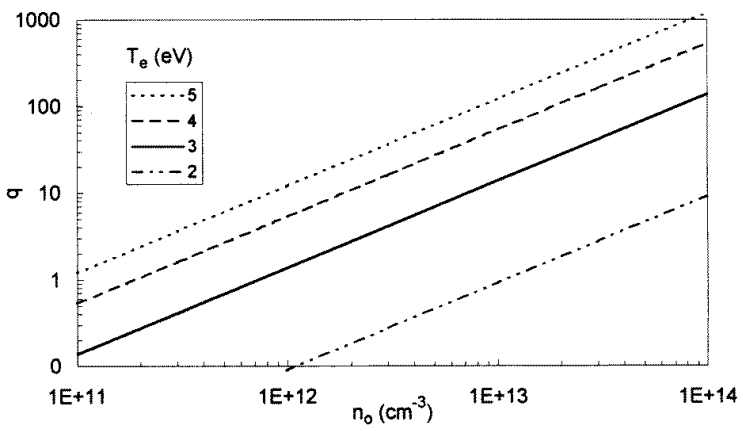

Figure 4. Dependence of the dimensionless parameter $q$ on density and temperature, for $T_{i}=0.05 \mathrm{eV}, p_{0}=3 \mathrm{mTorr}$, $a=5 \mathrm{~cm}$.

where the constant $A$ is of order $10^{17} \mathrm{~cm}^{-1} \mathrm{~s}^{-1}$ (argon, $T_{i}=0.0257 \mathrm{eV}, T_{e}=3 \mathrm{eV}$ ). Thus, $D$ varies as $1 / n$, rather than as $n$, as for perpendicular diffusion of a fully ionized and fully magnetized plasma [3]. The steady-state equation of continuity is

$$
\nabla(D \nabla n)+Q=0 .
$$

Neglecting longitudinal ion diffusion in a long, thin cylinder, we have

$$
\frac{A}{r} \frac{\mathrm{d}}{\mathrm{d} r}\left(\frac{r}{n} \frac{\mathrm{d} n}{\mathrm{~d} r}\right)+Q=0
$$

\subsection{Case of $Q=$ constant}

If the source term is uniform, the finite solution of (9) is

$$
\ln n=C \frac{Q}{4 A} r^{2} \text {. }
$$

If we apply the boundary conditions $n=n_{0}$ at $r=0$, $n=\delta n_{0}$ at $r=a$, the density profile depends only on the edge density and is given by

$$
\frac{n}{n_{0}}=\delta^{r^{2} / a^{2}}
$$

Figure 3 shows such profiles for several values of $\delta$. Though these Gaussians are closer to figure 1 than Bessel functions, they are still not sufficiently 'triangular'. Uniform ionization can apply, for instance to discharges ionized by ultraviolet light or fast electron beams, such as in e-beam excited $\mathrm{CO}_{2}$ lasers.

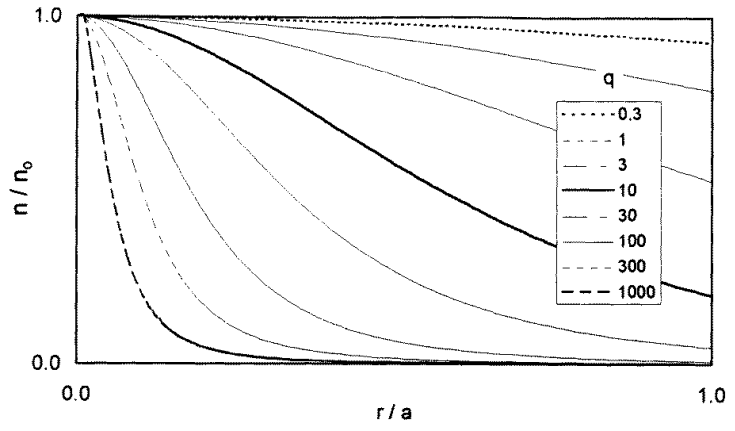

Figure 5. Computed density profiles for ionization proportional to density. The curves are in the same order as in the legend.

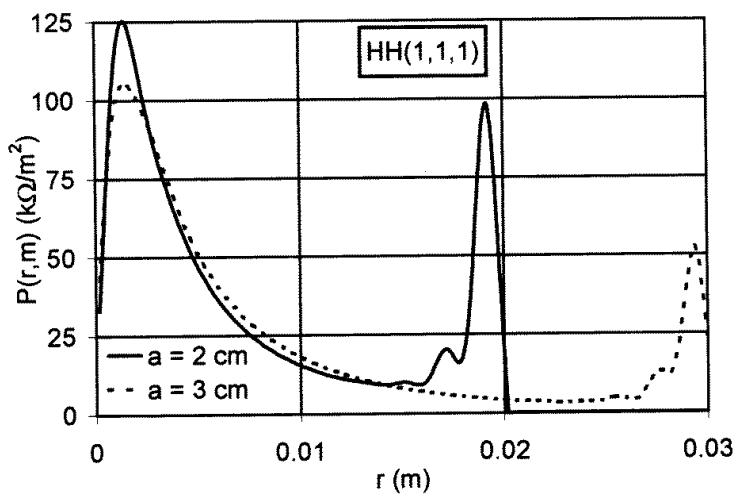

Figure 6. Plasma loading as a function of radius, computed for a half-wavelength $m=+1$ helical antenna and a triangular density profile. Other parameters were: $B_{0}=1 \mathrm{kG}, n=10^{13} \mathrm{~cm}^{-3}, p_{0}=3 \mathrm{mTorr}$ of argon and $f=27.12 \mathrm{MHz}[11]$.

\subsection{Case of $Q$ proportional to $n$}

The ionization frequency per unit volume is given by

$$
Q=n_{n} n_{e}\langle\sigma v\rangle_{i o n} \equiv G n_{e}
$$

where the ionization probability $\langle\sigma \nu\rangle_{i o n}$ is sensitive to $T_{e}$. (9) now becomes

$$
\frac{\mathrm{d}}{\mathrm{d} r}\left(\frac{r}{n} \frac{\mathrm{d} n}{\mathrm{~d} r}\right)=-\frac{G}{A} n r .
$$

Changing to dimensionless variables $n^{*}=n / n_{0}, r^{*}=r / a$, and $\left(^{\prime}\right)=a(\mathrm{~d} / \mathrm{d} r)$; defining

$$
q \equiv G n_{0} a^{2} / A
$$

and then dropping the $\left(^{*}\right)$, we obtain

$$
n^{\prime \prime}+\left(\frac{1}{r}-\frac{n^{\prime}}{n}\right) n^{\prime}+q n^{2}=0 .
$$

Values of $q$ for various temperatures and densities are shown in figure 4, and numerical solutions of (20) for various values of $q$ are shown in figure 5. These density profiles are 'triangular' only for intermediate values of $q$. The curve for $q=10$ resembles not only figure 1 but also profiles measured by L Porte [10] in an entirely different machine: $2.5 \mathrm{~cm}$ radius, $20 \mathrm{mTorr}$, and $800 \mathrm{G}$, with $T_{e}=3 \mathrm{eV}$ and peak density $\approx 3 \times 10^{13} \mathrm{~cm}^{-3}$, parameters for which $q=10$ is appropriate. 


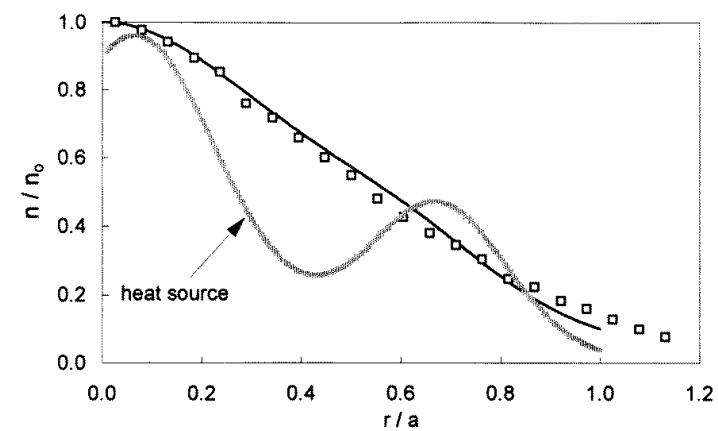

Figure 7. Density profile computed for a spatially varying source of plasma heating, as shown by the broad line. The points are the data of figure 1 .

\subsection{Case of bimodal energy deposition}

Numerical computations of energy deposition by coupled helicon and Trivelpiece-Gould (TG) waves [4] usually show two peaks, one near the surface due to TG absorption, and one near the axis due to helicon absorption. An example of such a bimodal energy deposition profile is shown in figure 6, calculated for a high-field discharge [11]. Local deposition of RF energy raises $T_{e}$ locally and increases $\langle\sigma \nu\rangle_{\text {ion }}$, and hence $q$ there. We have modelled this situation by using a function $q(r)$ consisting of two Gaussians centred at different radii. An example is shown in figure 7, where it is seen that the resulting profile $n(r)$ is 'triangular', resembling the measured data shown in figure 1 and reproduced in figure 7 .

\section{Summary}

Transverse diffusion of fully ionized plasmas in intermediate magnetic fields is shown to be governed by a diffusion coefficient inversely proportional to the density. The assumption of complete ionization is valid over a density range applicable to high-density plasma sources if the ion pumping effect is taken into account. This nonlinear diffusion, coupled with a physically reasonable RF absorption profile, can lead to the triangular density profiles observed experimentally in helicon discharges.

\section{References}

[1] Chevalier G and Chen F F 1993 J. Vac. Sci. Technol. A 11 1165

[2] Blackwell D D 1994 Master's Thesis UCLA. The data shown were taken more recently in the same machine.

[3] Chen F F Introduction to Plasma Physics and Controlled Fusion 2nd edn, vol 1 (New York: Plenum) ch 5

[4] Arnush D and Chen F F 1998 Phys. Plasmas 51239

[5] Sudit I D and Chen F F 1996 Plasma Sources Sci. Technol. 5 43, figure 10

[6] Book D L NRL Plasma Formulary (Washington, DC: Naval Research Laboratory) publication 0084-4040

[7] Sheldon J W 1962 Phys. Rev. Lett. 864

[8] McDaniel E W and Mason E A 1973 Mobility and Diffusion of Ions in Gases (New York: Wiley) p 274

[9] Gilland J, Breun R and Hershkowitz N 1998 Plasma Sources Sci. Technol. 7416

[10] Porte L 1997 Bull. Am. Phys. Soc. 421818

[11] Arnush D and Chen F F 1997 UCLA Report PPG-1573 